\title{
Solar-like oscillations in $\delta$ Scuti stars
}

\author{
R. Samadi ${ }^{1,2}$, M.-J. Goupil ${ }^{2}$, and G. Houdek ${ }^{3}$ \\ 1 Astronomy Unit, Queen Mary, University of London, London E14NS, UK \\ 2 Observatoire de Paris, LESIA, CNRS FRE 2461, 92195 Meudon, France \\ 3 Institute of Astronomy, University of Cambridge, Cambridge CB3 OHA, UK
}

Received 25 January 2002 / Accepted 5 September 2002

\begin{abstract}
Model computations of $\delta$ Scuti stars, located in the vicinity of the red edge of the classical instability strip, suggest amplitudes of solar-like oscillations larger than in cooler models located outside the instability strip. Solar-like amplitudes in our $\delta$ Scuti models are found to be large enough to be detectable with ground-based instruments provided they can be distinguished from the opacity-driven large-amplitude pulsations. There would be advantages in observing simultaneously opacity-driven and stochastically excited modes in the same star. We anticipate their possible detection in the context of the planned asteroseismic space missions, such as the French mission COROT (COnvection ROtation and planetary Transits). We propose known $\delta$ Scuti stars as potential candidates for the target selection of these upcoming space missions.
\end{abstract}

Key words. stars: interiors - stars: variables: $\delta \mathrm{Sct}$

\section{Introduction}

The $\delta$ Scuti stars are in general main sequence stars with masses between $1.5 M_{\odot}$ and $2.5 M_{\odot}$. They are located inside the classical instability strip (IS hereafter) where the $\kappa$-mechanism drives low-order radial and nonradial modes of low degree to measurable amplitudes (opacity-driven unstable modes). Only a small number of opacity-driven modes are observed in $\delta$ Scuti stars (for a review see e.g. Gautschy \& Saio 1996), but their amplitudes, which are limited by nonlinear processes, are much larger than stochastically driven intrinsically stable solar-like $p$ modes.

For main-sequence stars with surface convection zones, located outside the IS, model computations suggest all modes to be intrinsically stable but excited stochastically by turbulent convection; for models located near the red edge of the IS the predicted velocity amplitudes become as large as 15 times the solar value (Houdek et al. 1999). Moreover, these computations suggest that models located inside the IS can pulsate simultaneously with modes excited both by the $\kappa$-mechanism and by the turbulent velocity field.

Provided that many modes can be detected, highfrequency $\mathrm{p}$ modes are more easily identified than low frequency $\mathrm{p}$ modes. Hence there are advantages of observing simultaneously both types of modes in the same star. As a first step, high-frequency p modes can help to determine the fundamental stellar parameters (e.g., luminosity, effective temperature) more accurately, whereas low-frequency modes, which

Send offprint requests to: $\mathrm{R}$. Samadi, e-mail: Reza.Samadi@obspm.fr are strongly sensitive to the properties of the deep layers of the star, can then be used as a diagnostic for the inner properties of the model. Such developments are outside the scope of the present paper and we only outline briefly the underlying idea.

The nearly regular frequency spacing of solar-like modes of high order (i.e., the large frequency separation) depends predominantly on the structure of the surface layers and consequently provides further constraints on the equilibrium models. Their degree $l$ and azimuthal order $m$ can be identified with the help of the classical echelle diagram method; this method was successfully tested by the simulation results of the COROT Seismic Working Group (Appourchaux 2002, personal communication); this severely constrains the fundamental stellar parameters (mass, age, chemical composition) of models for which the frequencies of computed oscillation modes are similar to the observed high-order modes (Berthomieu et al. 2002). Moreover, solar-type modes also provide information on the star's mean rotation rate.

A nearly regular spacing in frequency is also observed for opacity-driven low-frequency modes (Breger et al. 1999); the large separation of these low-frequency modes has to be similar between observations and theoretical models which satisfy also the properties of the observed high-frequency solartype $\mathrm{p}$ modes. However, some of the opacity-driven modes deviate from the mean value of the large frequency separation; these modes are so-called mixed modes which provide details of the stellar core and of the precise evolutionary stage of the observed star (Unno et al. 1989 and references therein). This deviation from the mean value of the large frequency separation could suggest the presence of mixed modes. The problem 
is further complicated by the fact that the rotational splitting frequency components are no longer equidistant for these fast rotators, i.e. these frequencies could erroneously be identified as frequencies of mixed modes. However, knowing the mean rotation rate from the high-frequency splittings of solar-type $\mathrm{p}$ modes, the frequency splittings of the low-frequency opacitydriven modes can be computed in the manner of Dziembowski \& Goode (1992) (see also Soufi et al. 1998).

The understanding of the physics responsible for the return to stability of opacity-driven modes at the red edge of the IS is still in its infancy. As the star becomes cooler the extent of the surface convection zone increases, thereby making the effect of convection-pulsation coupling on mode stability progressively more important. Several authors have tried to model the location of the red edge, e.g., Baker \& Gough (1979), Bono et al. (1995) for RR Lyrae stars and e.g., Houdek (1996) and Xiong $\&$ Deng (2001) for $\delta$ Scuti stars. Although the authors assumed various models for the time-dependent treatment of convection, they all concluded that convection dynamics crucially effect the location of the red edge; however, different results were reported as to whether the convective heat flux (e.g., Bono et al. 1995), the momentum flux (e.g., Houdek 1996) or turbulent viscosity (Xiong \& Deng 2001) is the crucial agent for stabilizing the modes at the red edge. In all these investigations, the predicted position of the red edge depends crucially on the assumed convection parameters, such as the mixing-length parameter or whether acoustic emission is included or neglected in the equilibrium model (Houdek 2000).

Although it is possible from Fig. 13 of Houdek et al. (1999) to conclude that both types of modes can be excited simultaneously in the same star, amplitudes of stochastically excited modes for stars located inside the instability strip were not explicitly carried out by Houdek et al. (1999) and their possible detection were not addressed.

The aim of this paper is to demonstrate that models of stars, located inside the IS and near the red edge, can exhibit both opacity driven modes and solar-like oscillations with sufficiently large amplitudes to be detectable with today's groundbased instruments. Consequently the planned asteroseismology space missions, such as COROT (COnvection ROtation and planetary Transits, Baglin \& The Corot Team 1998) or Eddington (Favata et al. 2000), will detect these oscillations even more easily.

Section 2 describes the equilibrium models, and the linear analysis results are discussed in Sect. 3, which are obtained from solving the equations of linear nonadiabatic oscillations in which convection is treated with the time-dependent, nonlocal formalism by Gough (1976, 1977, hereafter G'MLT). Furthermore, the effect of acoustic radiation in the equilibrium model on the stability properties is taken into account in the manner of Houdek (2000, and references therein). In this paper we consider only radial $\mathrm{p}$ modes.

Amplitudes of solar-like oscillations result from the balance between damping and stochastic driving by turbulence. The rate at which the turbulence injects energy into the p modes is estimated in the manner of Samadi \& Goupil (2001, Paper I hereafter) and is discussed in Sect. 4.
Table 1. Stellar parameters for the envelope models A1, A2, B1, B2 and $\mathrm{C} ; R$ is the stellar radius at the photosphere $\left(T=T_{\mathrm{eff}}\right)$, and $v_{\mathrm{c}}$ is the acoustic cut-off frequency.

\begin{tabular}{cccccc}
\hline \hline Model & $\begin{array}{c}T_{\text {eff }} \\
{[\mathrm{K}]}\end{array}$ & $(b-y)_{0}$ & $\begin{array}{c}R \\
{\left[R_{\odot}\right]}\end{array}$ & $\begin{array}{c}v_{\mathrm{c}} \\
{[\mathrm{mHz}]}\end{array}$ & $\begin{array}{c}\text { acoustic } \\
\text { radiation }\end{array}$ \\
\hline $\mathrm{A} 1, \mathrm{~A} 2$ & 6839 & 0.235 & 2.40 & 1.4 & included \\
$\mathrm{B} 1, \mathrm{~B} 2$ & 6839 & 0.235 & 2.40 & 1.4 & neglected \\
$\mathrm{C}$ & 6650 & 0.262 & 2.54 & 1.3 & neglected \\
\hline
\end{tabular}

Table 2. Acoustic emissivity coefficient $\Lambda$ and Mach-number dependence $\Gamma$ assumed in the acoustic radiation model for the stellar models A1 and A2.

\begin{tabular}{crl}
\hline \hline Model & \multicolumn{1}{c}{$\Lambda$} & \multicolumn{1}{c}{$\Gamma$} \\
\hline A1 & 100 & 5 \\
A2 & 2000 & 7.5 \\
\hline
\end{tabular}

In Sect. 5 we address the possibilities and conditions for detecting solar-type oscillations in $\delta$ Scuti stars with groundbased instruments and propose possible candidates, some of which are listed in the catalogue by Rodríguez et al. (2000). Conclusions are given in Sect. 6.

\section{The stellar models}

Equilibrium envelope models are computed in the manner of Houdek et al. (1999) using G'MLT formulation for convection. Integration starts at an optical depth of $\tau=10^{-4}$ and ends at a radius fraction 0.2. Radiation is treated in the Eddington approximation and the atmosphere is assumed to be grey and plane parallel. In G'MLT formulation two more parameters, $a$ and $b$, are introduced which control the spatial coherence of the ensemble of eddies contributing to the total heat and momentum fluxes $(a)$, and the degree to which the turbulent fluxes are coupled to the local stratification $(b)$. In this paper we choose $a^{2}=900$ and $b^{2}=2000$ in order to obtain stable modes in the frequency range in which the damping rates exhibit a local minimum (e.g., at about $1.1 \mathrm{mHz}$ for model $\mathrm{C}$; see Sect. 3 and Fig. 2). The mixing-length parameter $\alpha$ has been calibrated to a solar model to obtain the helioseismically inferred depth of the convection zone of 0.287 of the solar radius (Christensen-Dalsgaard et al. 1991).

All models assume solar chemical composition and have mass $M=1.68 M_{\odot}$ and luminosity $L=11.3 L_{\odot}$, but differ in effective temperature $T_{\text {eff }}$, and whether or not acoustic radiation is included in the equilibrium computations. Table 1 lists the fundamental stellar parameters of these models. The models A1, A2 , B1 and B2 are hotter than model C and are located inside the IS and close to the red edge. Models A 1 and $\mathrm{A} 2$ differ from models B1 and B2 by the inclusion of acoustic radiation by turbulence in the envelope calculations. In this model for acoustic radiation in the equilibrium model two more parameters are introduced (Houdek \& Gough 1998): the emissivity coefficient $\Lambda$ and the parameter $\Gamma$ which describes the powerlaw dependence of the acoustic power emission on the turbulent Mach number. A Mach-number dependence of $\Gamma=5$ assumes that acoustic emission is dominated by the energy-bearing 
eddies; if acoustic emission is predominantly emitted by inertial-range eddies $\Gamma$ has the value 7.5. Table 2 lists the values of $\Lambda$ and $\Gamma$ that are assumed in the models A1 and A2. The values for $\Lambda$ provide for a solar model a similar value for the acoustic flux $F_{\text {ac }}$ as the estimates of Stein (1968) and Musielak et al. (1994). For all the models, except for model B2, we assume for the mixing-length parameter the calibrated solar value $\alpha=2.037$; for model B2 the value $\alpha=1.5$ is assumed.

Figure 1 displays the locations of these models in the colour-magnitude diagram. Evolutionary tracks (dashed curves) are shown for models with various masses and are obtained with the CESAM code by Morel (1997) as described by Samadi et al. (2001a). The transformation from luminosity, effective temperature and surface gravity to absolute magnitude $M_{v}$ and dereddened colour indices $(b-y)_{\mathrm{o}}$ are obtained from the Basel Stellar Library (Lejeune et al. 1998). The blue and red edges of the fundamental radial modes (solid curves) are calculated in the manner of Houdek et al. (1999). The positions of the observed $\delta$ Scuti stars (filled circles) are taken from Rodríguez et al. (2000): absolute magnitudes, derived from Hipparcos distances and dereddened colour indices were kindly supplied by E. Rodríguez (2001, personal communication; see Rodríguez \& Breger 2001, for details).

\section{Stability analysis}

The stability computations are as described in Houdek (2000, and references therein). In particular they include the Lagrangian perturbations of the turbulent fluxes (heat and momentum) according to Gough's (1977) time-dependent formulation. Assuming a temporal dependence, $\exp (-\mathrm{i} \omega t)$, for the pulsations, the complex eigenfrequencies of the modes can be written as $\omega=\omega_{\mathrm{r}}+\mathrm{i} \omega_{\mathrm{i}}$, which defines the cyclic pulsation frequency $v=\omega_{\mathrm{r}} / 2 \pi$ and the damping/growth rate $\eta=-\omega_{\mathrm{i}} / 2 \pi$. The outer boundary conditions are applied at the temperature minimum, the mechanical boundary condition being consistent with a perfectly reflecting surface; at the base of the envelope, conditions of adiabaticity and vanishing displacement are imposed.

For model $\mathrm{C}$ all the modes are found to be linearly stable (i.e., $\eta>0$ ) as is expected for models lying well outside the IS. This is also found for the hotter model B1. For the model A1 (resp. A2) the first four (resp. three) radial modes, $n=1, \ldots, 4$ (resp. $n=1,2,3$ ), are found to be overstable. With the inclusion of a model for the acoustic radiation in the equilibrium structure the efficacy with which convection transports the turbulent fluxes is decreased (see Houdek \& Gough 1998). This leads to a decrease in the turbulent Mach number and to a consequent reduction of the stabilizing influence of the perturbed momentum flux on the mode damping. The driving eventually dominates over the damping leading to overstable modes.

Reducing $\alpha$ has a similar effect on mode stability than the inclusion of acoustic radiation in the equilibrium model (see Houdek \& Gough 1998; Michel et al. 1998; Houdek 2000). The model B2 was computed with the smaller mixing-length parameter $\alpha=1.5$, leading to overstable modes with radial orders $n=1,2,3$.
Table 3. Frequency $v$, damping/growth rate $\eta$ and stability coefficient $\omega_{\mathrm{i}} / \omega_{\mathrm{r}}$ for all overstable radial $\mathrm{p}$ modes predicted for the models $\mathrm{A} 1$, $\mathrm{A} 2$ and $\mathrm{B} 2$.

\begin{tabular}{ccccc}
\hline \hline Model & $n$ & $\begin{array}{c}v \\
{[\mu \mathrm{Hz}]}\end{array}$ & $\begin{array}{c}\eta \\
{[\mathrm{nHz}]}\end{array}$ & $\begin{array}{c}\omega_{\mathrm{i}} / \omega_{\mathrm{r}} \\
\times 10^{-6}\end{array}$ \\
\hline \multirow{3}{*}{$\mathrm{A} 1$} & 1 & 123 & -0.03 & 0.25 \\
& 2 & 161 & -0.31 & 1.92 \\
& 3 & 202 & -4.14 & 20.48 \\
& 4 & 244 & -3.90 & 15.97 \\
& 1 & 124 & -0.04 & 0.36 \\
$\mathrm{~A} 2$ & 2 & 162 & -0.40 & 2.47 \\
& 3 & 203 & -1.27 & 6.25 \\
& 1 & 124 & -0.04 & 0.34 \\
$\mathrm{~B} 2$ & 2 & 161 & -0.31 & 1.95 \\
& 3 & 203 & -0.83 & 4.09 \\
\hline
\end{tabular}

Table 3 displays the frequency $v$ and damping/growth rate $\eta$ for all overstable radial modes $(\eta<0)$ found in the models $\mathrm{A} 1$, A2 and B2.

Figure 2 displays the damping rates as function of frequency for all stable modes and for all stellar models. The coolest model $\mathrm{C}$ exhibits a sharp dip in $\eta$ at about $1.1 \mathrm{mHz}$, which we moderated by applying a median filter with a width in frequency corresponding to nine radial modes; the result is plotted by the long-dashed curve in Fig. 2. This pronounced depression in $\eta$ in model $\mathrm{C}$ is related to the structure of the outer superadiabatic boundary layer: with decreasing surface temperature the location of the superadiabatic boundary layer is shifted progressively deeper into the star. This modifies the thermodynamic properties of this boundary layer of finite thickness, in particular, the thermal relaxation time (Balmforth 1992). The thermodynamic coupling between the pulsations and the superadiabatic boundary layer becomes more efficient in cooler models, thereby promoting the depression in the damping rates by radiative processes (see Houdek et al. 1999).

\section{Excitation rate and amplitude spectrum}

The rms value of the mode surface velocity, $v_{\mathrm{s}}$, is related to the damping rate, $\eta$, and to the rate at which energy is injected into the mode (excitation rate), $P$, by

$v_{\mathrm{s}}^{2}=\xi_{\mathrm{r}}^{2}\left(r_{\mathrm{s}}\right) \frac{P}{2 \eta I}$

where $\xi_{\mathrm{r}}$ is the radial displacement eigenfunction, $r_{\mathrm{s}}$ is the radius at which the surface velocities are measured and which we assume to be $200 \mathrm{~km}$ above $T_{\text {eff }}$, and the mode inertia $I$ satisfies $I=\int_{0}^{M} \boldsymbol{\xi}^{*} \cdot \boldsymbol{\xi} \mathrm{d} m$

The rate of energy injected into a mode is computed according to Paper I and is proportional to

$P(\omega) \propto \int_{0}^{M} \rho w^{3} \ell^{4}\left(\frac{\mathrm{d} \xi_{\mathrm{r}}}{\mathrm{d} r}\right)^{2} \mathcal{S}(\omega, m) \mathrm{d} m$,

where $\rho$ is the density, $\ell$ is the mixing length, and $w$ is the vertical component of the rms velocity of the convective elements. The function $\mathcal{S}(\omega, m)$ describes approximately contributions from eddies with different sizes to the excitation rate $P$. 


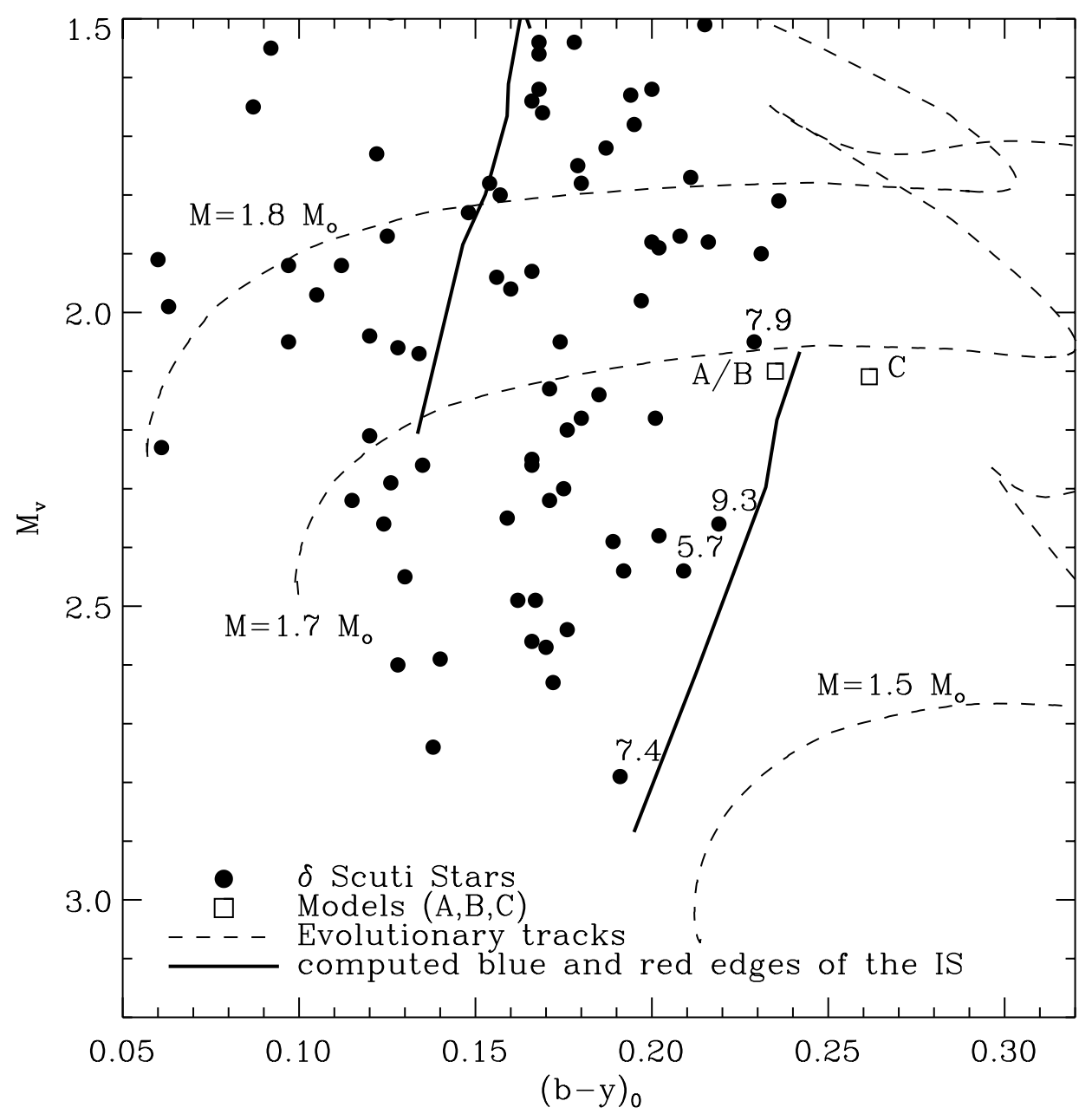

Fig. 1. Colour-magnitude diagram: filled circles display the positions of observed $\delta$ Scuti stars from the Rodríguez et al. (2000) catalogue. Squares indicate the positions of the models A1, A2, B1, B2 and C (see Table 1). Dashed curves show evolutionary tracks for models with masses $1.5 M_{\odot}, 1.7 M_{\odot}$ and $1.8 M_{\odot}$. Solid curves display theoretical locations of the blue and red edges for the fundamental radial modes according to Houdek et al. (1999). Numbers associated with the symbols indicate apparent magnitudes $V$ for selected observed $\delta$ Scuti stars.

Detailed expressions for $\mathcal{S}(\omega, m)$ were given in Paper I. For estimating $\mathcal{S}$, assumptions for the turbulent kinetic energy spectrum $E(k)$, and for the turbulent spectrum of the entropy fluctuations $E_{\mathrm{s}}(k)$ have to be made, where $k$ is the eddy wavenumber. In this paper we assume for $E(k)$ the "Nesis Kolmogorov Spectrum" (NKS hereafter) as discussed in Paper I. This turbulent spectrum is obtained from observations of the solar granulation by Nesis et al. (1993), and leads to the best agreement between a solar model using our stochastic excitation theory and solar measurements (Samadi et al. 2001b).

Results for the estimated excitation rate $P$ are depicted in Fig. 3. For the models $\mathrm{A} 1, \mathrm{~A} 2$ and $\mathrm{B} 1$ the excitation rate $P$ is about one magnitude larger than for model $\mathrm{C}$. This is a result of the larger convective velocities in the superadiabatic boundary layers of the models A1, A2 and B1 (see Fig. 4), which are all hotter than model $\mathrm{C}$. The frequency dependence of $P$ for the models A1 and B1 are similar as it is also the case for the models A2 and B2; the latter two models, however, are quite different from the first two models. This difference in the frequency dependence of $P$ is a consequence of the different profiles of the convective velocities $w$ (see Fig. 4); the profiles of $w$ are similar for A2 and B2 but differ substantially from A1 and B1.
For the models A2 and B2 the efficacy of convection has been reduced severely by either including acoustic radiation in the equilibrium model (A2) or by reducing the mixing-length parameter $\alpha$ to a value much smaller than the calibrated value for a solar model (B2). This results in shallower superadiabatic regions and in larger superadiabatic temperature gradients; pulsation modes in $\mathrm{A} 2$ and $\mathrm{B} 2$ are therefore predominantly excited at the very top of the convection zone, whereas in the models $\mathrm{A} 1$ and $\mathrm{B} 1$ the modes are excited over a larger driving region. The two sets of values for $\Lambda$ and $\Gamma$, listed in Table 2, provide in a solar model approximately the same value for the acoustic flux $F_{\text {ac }}$ (see Sect. 2). In model A2 (which assumes $\Lambda=2000$ and $\Gamma=7.5)$, however, $F_{\text {ac }}$ is about three times larger than in model A1 (which assumes $\Lambda=100$ and $\Gamma=5$ ), and the associated velocities, plotted in Fig. 4, are correspondingly greater.

It is interesting to note that in Fig. 4 the convective velocities in the superadiabatic boundary layers of models A1 and A2 are somewhat larger than the others. Although including acoustic radiation in the mean stellar structure leads to a reduction of the maximum turbulent Mach number $M_{\mathrm{t}} \equiv w / c$ (by $\sim 1.5 \%$ for model $\mathrm{A} 1$ and $\sim 5 \%$ for model $\mathrm{A} 2$ relative to $\mathrm{B} 1$ ), the whole structure of the outer layers changes too, thereby increasing 


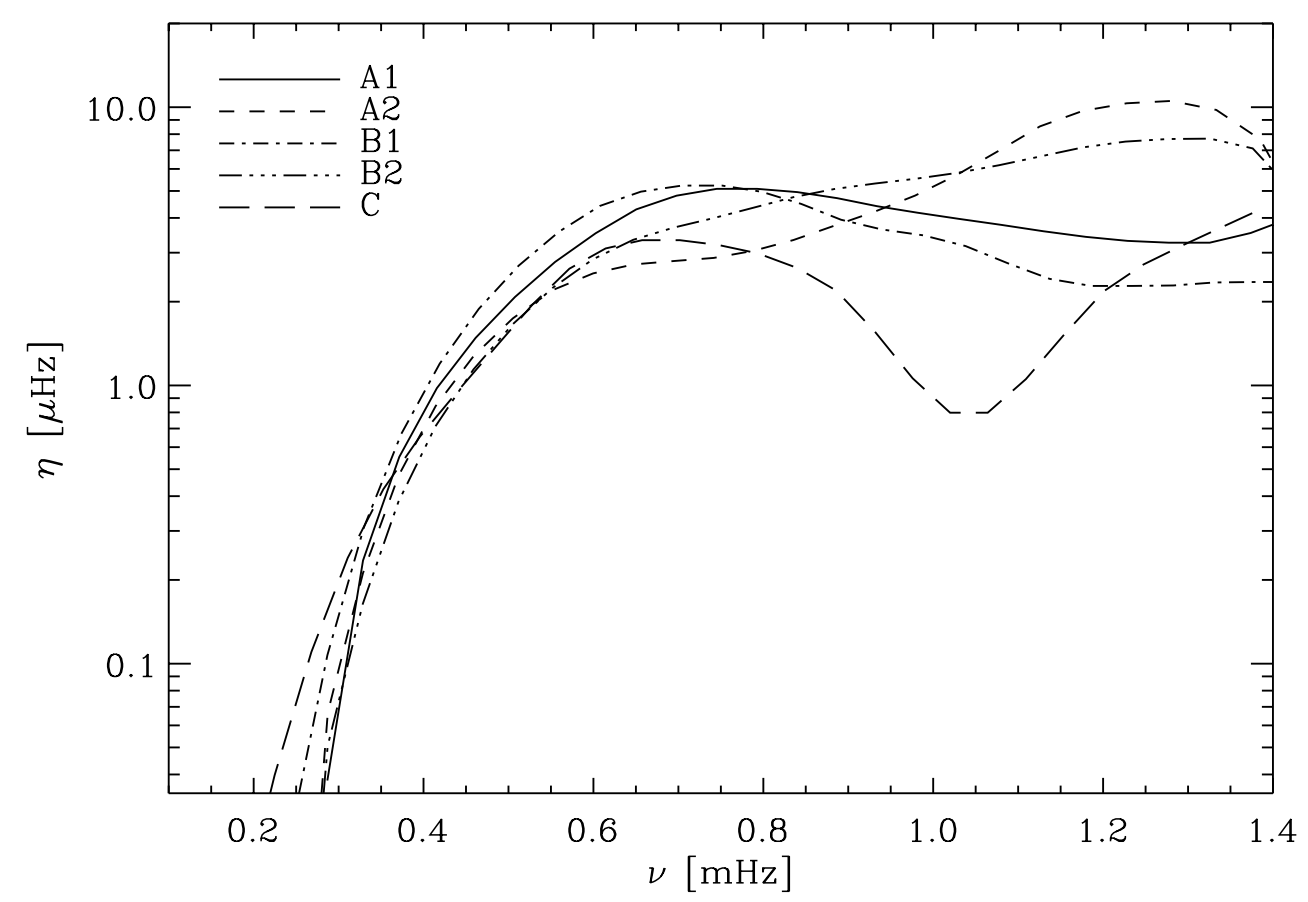

Fig. 2. Damping rates of stable radial p modes as function of frequency for all stellar models.

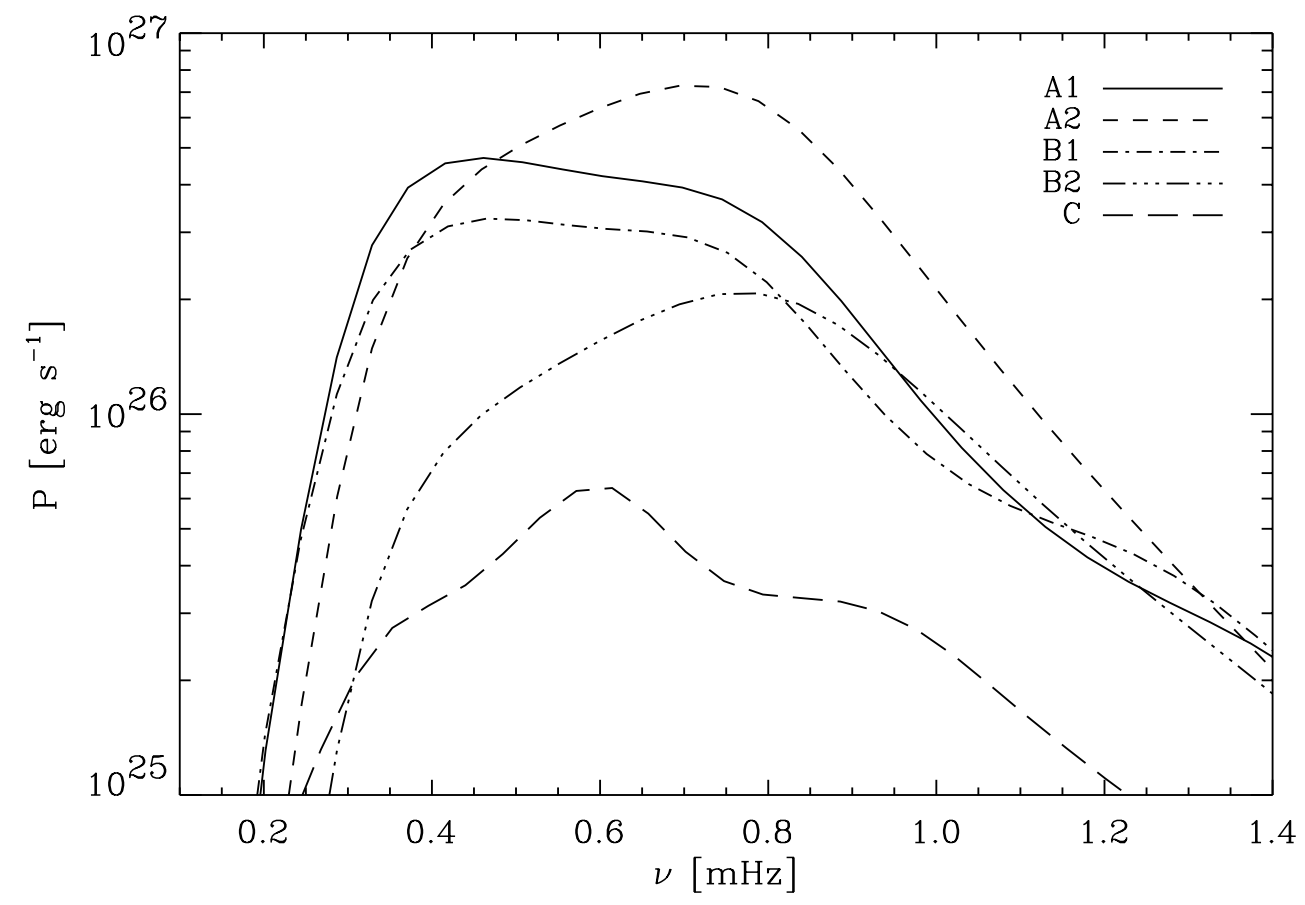

Fig. 3. Excitation rate $P$ as function of frequency for all stellar models.

the locally defined adiabatic sound speed $c$. This leads to an increase of the convective velocities $w$ in the very outer layers despite the decrease in $M_{\mathrm{t}}$.

The less smooth frequency dependence (wiggles) of $P$ for $v \gtrsim 0.7 \mu \mathrm{Hz}$ for model B1 (and model C) is related to the location and extent of the driving region: the radial eigenfunctions $\xi_{\mathrm{r}}$ vary rapidly with depth and frequency (particularly for the high-frequency modes). As discussed above, in the models A2 and B2 the modes are predominantly excited in a shallow region beneath the surface, where the expression $\left(\mathrm{d} \xi_{\mathrm{r}} / \mathrm{d} r\right)^{2}$ (see
Eq. (3)) varies monotonically with frequency (Goldreich et al. 1994; Samadi et al. 2002), leading to the smooth frequency dependence of $P$ for $\nu \gtrsim 0.7 \mu \mathrm{Hz}$, as depicted in Fig. 3. The larger driving regions in the models $\mathrm{B} 1$ and $\mathrm{C}$ extend to layers where the expression $\left(\mathrm{d} \xi_{\mathrm{r}} / \mathrm{d} r\right)^{2}$ no longer varies monotonically with frequency leading to the frequency-dependence of $P$ as shown by the dot-dashed and long-dashed curves in Fig. 3.

In the top panel of Fig. 5 the surface velocity amplitudes $v_{\mathrm{s}}$ are depicted for all stellar models, computed according to Eq. (1). In the models A1, A2, B1 and B2 the amplitudes of 


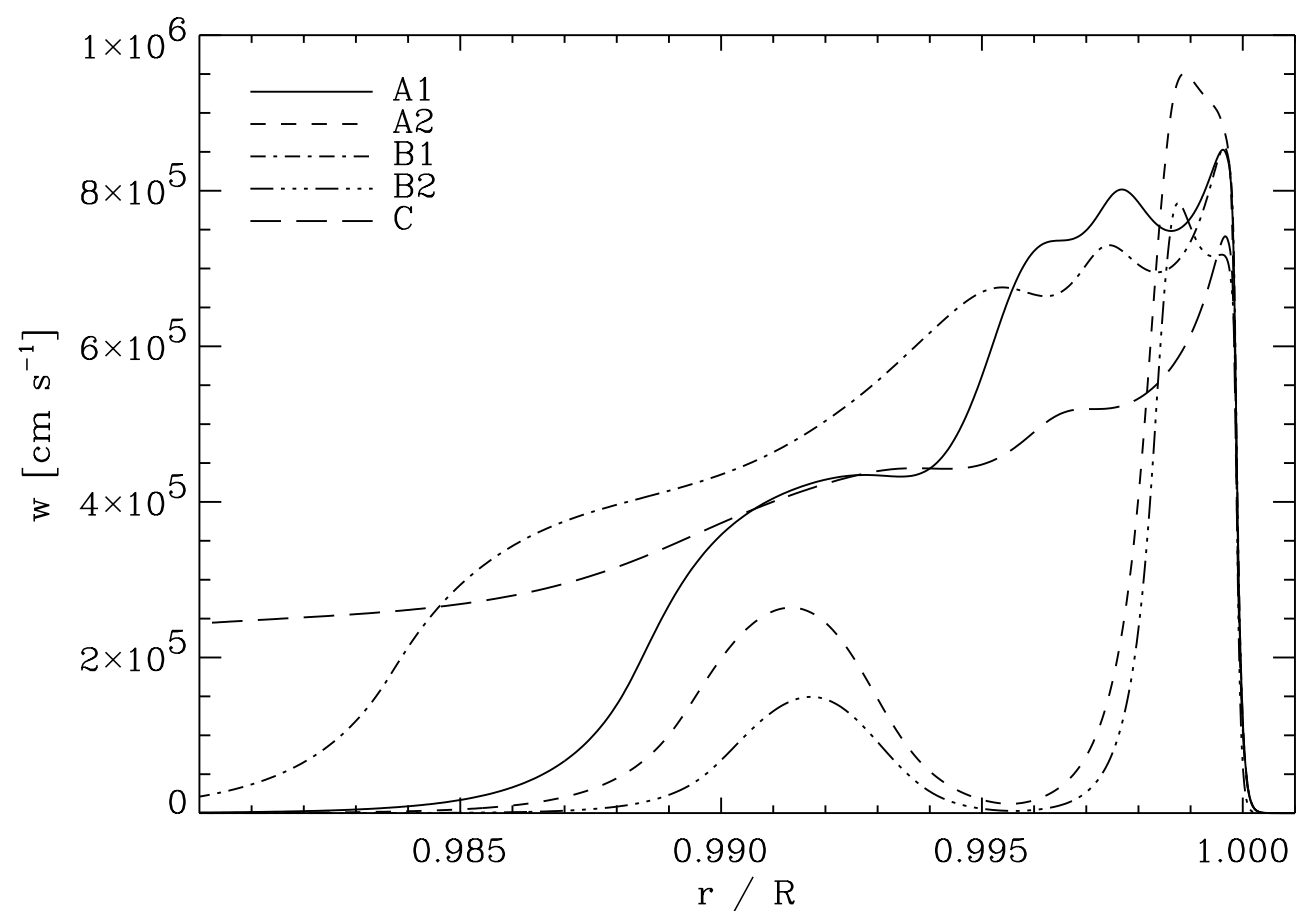

Fig. 4. Depth dependence of the vertical component of the convective velocity, with $R$ being the radius at the photosphere $\left(T=T_{\text {eff }}\right)$.

stochastically excited $\mathrm{p}$ modes are larger $\left(\sim 5-9 \mathrm{~ms}^{-1}\right)$ than in model C $\left(\sim 2 \mathrm{~ms}^{-1}\right)$.

For estimating the luminosity amplitudes the full nonadiabatic luminosity eigenfunctions have to be used. The relative luminosity amplitudes, $\delta L / L$, are linearly related to the velocity amplitudes, i.e. they are proportional to the ratio of the luminosity eigenfunction over the displacement eigenfunction. This ratio is determined by the solution of the nonadiabatic pulsation equations and is independent of a stochastic excitation model (see Houdek et al. 1999). In the middle panel of Fig. 5 the amplitude ratios, $\Delta L / \Delta v_{\mathrm{s}}$, are plotted as a function of frequency for all stellar models. The shape of the amplitude ratios are in general similar between all the models with the smallest ratios predicted for the models A2 and A1. Only at the highest frequencies the amplitude ratios are considerably larger in A2 and $\mathrm{B} 2$; at high frequencies nonadiabatic effects due to radiative dissipation in the radiative zone, below the shallow surface convection zones in A2 and B2, lead to an increase in the amplitude of the luminosity eigenfunctions and consequently in the luminosity amplitudes. The velocity amplitudes in Fig. 5 are obtained $200 \mathrm{~km}$ above the photosphere $\left(T=T_{\text {eff }}\right)$ and do increase by a factor of about two at the outermost meshpoint of the model, i.e. at an optical depth $\tau=10^{-4}$.

We predict a maximum value of the luminosity amplitude $\delta L / L \sim 97 \mathrm{ppm}$ for model A1, $\delta L / L \sim 150 \mathrm{ppm}$ for model A2, $\delta L / L \sim 101 \mathrm{ppm}$ for model B1, $\delta L / L \sim 98 \mathrm{ppm}$ for model B2 and $\delta L / L \sim 84 \mathrm{ppm}$ for model $\mathrm{C}$. These results are summarized in Table 4.

The dotted horizontal line in the middle panel of Fig. 5 represents an order-of-magnitude estimate of the amplitude ratio according to Kjeldsen \& Bedding (1995):

$\delta L / L \propto v_{\mathrm{s}} T_{\mathrm{eff}}^{-1 / 2}$
Kjeldsen \& Bedding derived this expression for a purely radiative model assuming simplified proportional relations in the adiabatic approximation. This simplified scaling law suggests smaller values for the amplitude ratios and consequently leads to smaller luminosity amplitudes $\delta L / L$, particularly at high frequencies, where nonadiabatic effects are important. At a frequency $v \simeq 1 \mathrm{mHz}$, for example, the scaling law (4) predicts for model A1 a luminosity amplitude which is about three times smaller than that obtained from the nonadiabatic computation.

There is evidence that energy equipartition holds for the Sun (apparently fortuitously); an estimate of the total energy in the modes is, however, only possible for the Sun for which accurate data are available; using GONG data the total energy for modes with degrees $l=0, \ldots, 300$ and with radial order $n$ up to 10 is found to be approximately $2 \times 10^{6} E_{0}$, where $E_{0} \sim 2 \times 10^{28}$ erg is the maximum value of the kinetic energy in a particular ridge (i.e. for a particular value of $n$ ) and which is independent of $l$ (see Fig. 5 of Komm et al. 2000). The value $2 \times 10^{6}$ is also roughly equal to the number of granules on the solar surface, a result which supports the energy equipartition principle. In other stars, however, energy equipartition does not necessarily hold, because we have a nonequilibrium dynamical (yet statistically steady) system in which the damping and excitation is balanced in a nonlinear way by the energy input and output, i.e. it is not determined by equilibrium. In such a nonequilibrium situation there is no general physical principle limiting the ratio of the energy in the oscillation mode to the energy in the convection. Another facet of such a dynamical process is provided by the reaction of convection to the acoustical radiation; although the latter contributes towards augmenting the damping of an eddy, the resultant change of the background stratification is such as to augment the driving by even more, causing the convective velocities to increase. 
Table 4. Maximum values of the estimated velocity, $v_{\mathrm{s}}$, and luminosity, $\delta L / L$, amplitudes.

\begin{tabular}{ccc}
\hline \hline Model & $\begin{array}{c}v_{\mathrm{s}} \\
{\left[\mathrm{ms}^{-1}\right]}\end{array}$ & $\begin{array}{c}\delta L / L \\
{[\mathrm{ppm}]}\end{array}$ \\
\hline $\mathrm{A} 2$ & 8.6 & 150 \\
$\mathrm{~A} 1$ & 7.9 & 97 \\
$\mathrm{~B} 1$ & 4.9 & 101 \\
$\mathrm{~B} 2$ & 5.5 & 98 \\
$\mathrm{C}$ & 2.0 & 84 \\
\hline
\end{tabular}

It is perhaps interesting to mention that the acoustic energy flux generated by the fluctuating Reynolds stress of the turbulent velocity field is relatively small compared to the total energy flux carried by the convection; the ratio between the acoustic energy flux emitted by the energy-bearing eddies and the convective energy flux is proportional to $M_{\mathrm{t}}^{\Gamma}$ with $\Gamma=5$ for homogeneous, isotropic turbulence (see Lighthill 1952). The turbulent Mach number in $\delta$ Scuti stars is in general much smaller than unity and consequently this ratio is small. For the Sun this ratio is of the order of $\sim 10^{-3}$ (see e.g., Stein 1968). The acoustic flux emitted predominantly by inertial-range eddies is proportional to $M_{\mathrm{t}}^{\Gamma}$ with $\Gamma=15 / 2$ (see Goldreich \& Kumar 1990), i.e. it scales with an even higher power of the Mach number (see also Sect. 2). Consequently the total amount of acoustic energy injected into the $p$ modes is small compared to the energy carried by the convection. In a fully convective envelope the total energy flux (luminosity) is carried solely by the turbulent velocity field, i.e. in that case the luminosity is a measure of the total energy in the convection. Therefore the ratio between the energy supply rate for a particular mode and the luminosity, $P / L$, is proportional to the ratio between the energy in that mode and the total energy in the convection. In the Sun this ratio is of the order of $\sim 10^{-11}$ for the mode with the largest amplitude. In model A2 this ratio is $\sim 10^{-8}$, which is still small.

\section{Observational constraints for detecting solar-type oscillations}

There have been recent reports on the possible detection of solar-type oscillations in $\alpha$ Cen (HD 128620) by Bouchy \& Carrier (2001), in $\beta$ Hydri (HD 2151) by Bedding et al. (2001) and in Procyon A (HD 61421) by Martic et al. (1999; see also Barban et al. 1999), who obtained spectroscopic surface velocity measurements of these bright stars (the apparent magnitude $V=2.80$ for $\beta$ Hydri, $V=0.34$ for Procyon and $V=-0.1$ for $\alpha$ Cen) from the ground. The maximum values of the observed peak-velocity amplitudes are of the order $\sim 35 \mathrm{~cm} \mathrm{~s}^{-1}$ for $\alpha$ Cen, $\sim 50 \mathrm{~cm} \mathrm{~s}^{-1}$ for $\beta$ Hydri and $\sim 50 \mathrm{~cm} \mathrm{~s}^{-1}$ for Procyon. Current ground-based instruments are able to detect oscillations with velocity amplitudes of the order predicted for our models A1, A2 and B1, B2, but only for stars with an apparent magnitude $V$ of less than $\sim 3-4$ (Bouchy, personal communication). The HARPS (High-Accuracy Radial-velocity Planetary Search) project (Bouchy \& Carrier 2001), for example, will be able to detect oscillations with our predicted velocity amplitudes for stars with an apparent magnitude smaller than $\sim 4-5$.
This detection threshold is still too small for detecting solartype oscillations in $\delta$ Scuti stars located near the red edge of the IS, particularly in view of the fact that most of the currently known $\delta$ Scuti stars are even fainter. For example, the apparent magnitudes of known $\delta$ Scuti stars located nearest to the red edge (see Fig. 1) are between $V=5.7$ and $V=9.3$.

Future space missions with instruments dedicated to asteroseismology, however, will be able to detect solar-like oscillations in $\delta$ Scuti stars: the forthcoming space project COROT (Baglin \& The Corot Team 1998), for example, will reach a noise level of $0.7 \mathrm{ppm}$ (Auvergne \& the COROT Team 2000) for a star with an apparent magnitude of $V=6$, using photometric measurements. Therefore, in stars with similar magnitudes, COROT will be able to detect oscillation amplitudes as small as $\sim 3 \mathrm{ppm}$, a value which is similar to that measured in the Sun. The instrument on COROT will be limited by the photon noise only for stars with magnitudes larger than $V \simeq 9$ : i.e., for a star with magnitude $V \simeq 8$ the detection threshold will be $\sim 5 \mathrm{ppm}$. This threshold is small enough to detect and measure many solar-like oscillations in $\delta$ Scuti stars which are similar to the $\delta$ Scuti models considered in this paper.

\section{Conclusion}

We studied oscillation properties in $\delta$ Scuti stars located near the observed red edge of the classical instability strip. Such stars can pulsate with both opacity-driven modes and intrinsically stable stochastically driven (solar-like) modes. The estimated velocity amplitudes of the stochastically driven modes in our $\delta$ Scuti models are found to be larger than in cooler and pulsationally stable models lying outside the IS. This result supports the idea that solar-like oscillations in $\delta$ Scuti stars may be detected.

Including a model for the acoustic radiation in the equilibrium model results in a cooler red edge and does effect the properties of the excitation rate of p modes (see also Houdek \& Gough 1998; Houdek 2000); in particular the pulsation amplitudes do become larger and are predicted to be largest for a model with the largest acoustic flux $F_{\text {ac }}$ (i.e., model A2). Moreover, for the $\delta$ Scuti models considered in this paper, overstable modes were predicted only if either acoustic emission in the mean stratification was included or if the mixing-length parameter was reduced to a value smaller than suggested by a calibrated solar model.

A potential target star should neither be too cool (i.e., no opacity-driven modes) nor too hot (i.e., stochastically excited modes with amplitudes too small to be detectable). We quantify this with the illustrative case of our $\delta$ Scuti models with a mass $M=1.68 M_{\odot}$ and we identify the following $\delta$ Scuti stars from the Rodríguez et al. (2000) catalogue, located near the red edge, as potential candidates for the target selection of upcoming observing campaigns: HD57167, HD14147, HD208999 and HD105513.

Although the amplitudes of the solar-type oscillations, predicted in our $\delta$ Scuti models, are large enough to be detected from ground, today's ground-based instruments will detect such oscillations only in brighter $\delta$ Scuti stars with an apparent magnitude of up to $V \sim 3-4$ (Bouchy 2001, personal 

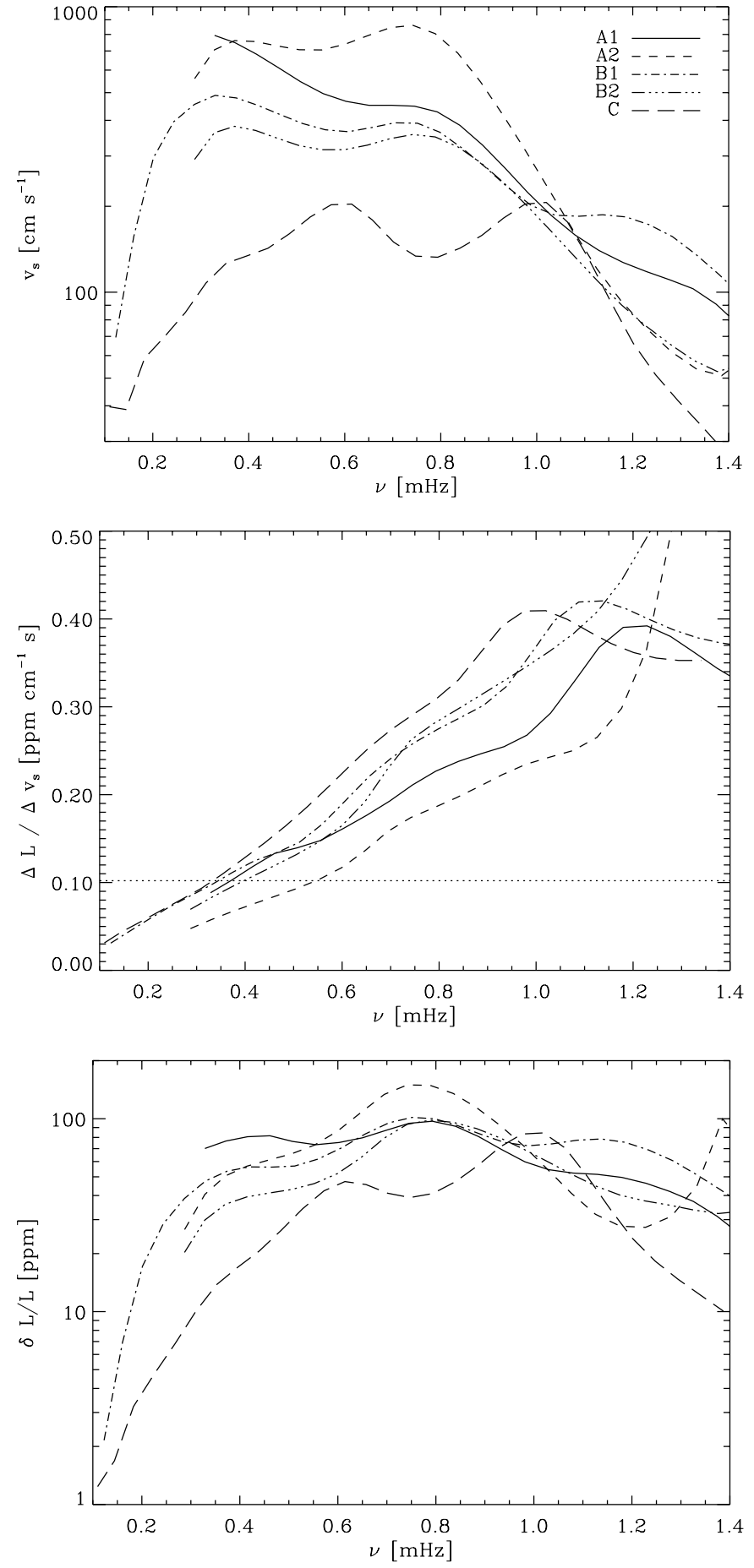

Fig. 5. Linear oscillation amplitudes of stable radial modes as a function of frequency. The top panel displays the surface velocity amplitudes and the middle panel the amplitude ratios, luminosity over velocity amplitudes, computed $200 \mathrm{~km}$ above the photosphere $(T=$ $\left.T_{\text {eff }}\right)$. The dotted horizontal line represents the results for Kjeldsen \& Bedding's (1995) scaling expression (4), assuming $T_{\text {eff }}=6839 \mathrm{~K}$. In the bottom panel the luminosity amplitudes are depicted. In the middle and bottom panel the luminosity amplitudes are computed at the outermost meshpoint of the models.

communication). However, new ground-based observing campaigns, such as the HARPS project (Bouchy \& Carrier 2001) will be able to detect stochastically excited oscillations in $\delta$ Scuti stars with an apparent magnitude of up to $V \sim 4-5$. Unfortunately, there are no such bright stars in the Rodríguez et al. (2000) catalogue which are located near the red edge, although some bright stars near the red edge may have opacitydriven modes with amplitudes too small to be detectable with today's ground-based instruments and are therefore not classified as $\delta$ Scuti stars.

The forthcoming space missions for asteroseismology, such as COROT (Baglin \& The Corot Team 1998) and Eddington (Favata et al. 2000) will be able to detect solar-like oscillations in faint $\delta$ Scuti stars. The large instrument on the Eddington spacecraft will measure stellar oscillations with amplitudes as small as $1.5 \mathrm{ppm}$ in stars with an apparent magnitude of $V \simeq 11$ assuming an observing period of 30 days. Moreover, Eddington's large field of view will allow it to monitor a large number of stars simultaneously. This will be helpful for detecting and classifying new $\delta$ Scuti stars and for measuring the location of the red edge of the IS with greater precision than it was possible before.

Acknowledgements. We thank E. Rodríguez for providing the $\delta$ Scuti data set in a convenient and immediate usable form, T. Lejeune for allowing us to use the Basel library and D. Cordier for providing it on the Internet. We thank A. Baglin for useful discussions on the COROT specifications, F. Bouchy for providing valuable information on the HARPS project and related experiments, and C. Catala and E. Michel for useful discussions on the possibilities of detecting new $\delta$ Scuti stars. We are grateful to Douglas Gough for very helpful discussions on stochastic mode excitation and to Mike Montgomery for improving the English. GH and RS acknowledge support by the Particle Physics and Astronomy Research Council of the UK. RS's work has been supported under the grant PPA/G/O/1998/00576.

\section{References}

Auvergne, M., \& the COROT Team 2000, in The Third MONS Workshop: Science Preparation and Target Selection, ed. T. C. Teixeira, T. Bedding (Aarhus University: Aarhus), 135

Baglin, A., \& The Corot Team 1998, in New Eyes to See Inside the Sun and Stars, ed. F.-L. Deubner, J. Christensen-Dalsgaard, \& D.W. Kurtz (Kluwer: Dordrecht), IAU Symp. 185, 301

Baker, N. H., \& Gough, D. O. 1979, ApJ, 234, 232

Balmforth, N. J. 1992, MNRAS, 255, 603

Barban, C., Michel, E., Martic, M., et al. 1999, A\&A, 350, 617

Bedding, T. R., Butler, R., Kjeldsen, H., et al. 2001, ApJ, 549, L105

Berthomieu and the COROT Seismic Working Group, in Proc. Asteroseismology Across the HR Diagram, 1-5 July 2002, Porto, in preparation

Bono, G., Caputo, F., Castellani, V., et al. 1995, ApJ, 442, 159

Bouchy, F., \& Carrier, F. 2001, A\&A, 374, L5

Breger, M., Pamyatnykh, A. A., Pikall, H., \& Garrido, R. 1999, A\&A, 341,151

Christensen-Dalsgaard, J., Gough, D. O., \& Thompson, M. J. 1991, ApJ, 378, 413

Dziembowski, W. A., \& Goode, P. R. 1992, ApJ, 394, 670

Favata, F., Roxburgh, I., \& Christensen-Dalsgaard, J. 2000, in The Third MONS Workshop: Science Preparation and Target Selection, ed. T. C. Teixeira, \& T. Bedding (Aarhus University: Aarhus), 49

Gautschy, A., \& Saio, H. 1996, ARA\&A, 34, 551

Goldreich, P., \& Kumar, P. 1990, ApJ, 363, 694 
Goldreich, P., Murray, N., \& Kumar, P. 1994, ApJ, 424, 466

Gough, D. 1976, in Lecture notes in physics, Problems of stellar convection, ed. E. Spiegel, \& J.-P. Zahn (Springer: Berlin), vol. 71, 15

Gough, D. O. 1977, ApJ, 214, 196

Houdek, G. 1996, Ph.D. Thesis, Institut für Astronomie, Wien

Houdek, G. 2000, in Delta Scuti and Related Stars, ed. M. Breger, \& M. H. Montgomery (ASP: San Francisco), ASP Conf. Ser., 210, 454

Houdek, G., \& Gough, D. O. 1998, in Proc. SOHO 6/GONG 98 Workshop, Structure and dynamics of the interior of the Sun and Sun-like stars, ed. S. G. Korzennik, \& A. Wilson (ESTEC: Noordwijk), ESA SP-418, vol. 2, 479

Houdek, G., Balmforth, N. J., Christensen-Dalsgaard, J., \& Gough, D. O. 1999, A\&A, 351, 582

Kjeldsen, H., \& Bedding, T. R. 1995, A\&A, 293, 87

Komm, R. W., Howe, R., \& Hill, F. 2000, ApJ, 543, 472

Lejeune, T., Cuisinier, F., \& Buser, R. 1998, A\&AS, 130, 65

Lighthill, M. J. 1952, Proc. R. Soc. Lond., A211, 564

Martic, M., Schmitt, J., Lebrun, J.-C., et al. 1999, A\&A, 351, 993

Michel, E., Hernández, M. M., Houdek, G., et al. 1999, A\&A, 342, 153
Morel, P. 1997, A\&AS, 124, 597

Musielak, Z. E., Rosner, R., Stein, R. F., \& Ulmschneider, P. 1994, ApJ, 423, 474

Nesis, A., Hanslmeier, A., Hammer, R., et al. 1993, A\&A, 279, 599

Rodríguez, E., \& Breger, M. 2001, A\&A, 366, 178

Rodríguez, E., López-González, M. J., \& López de Coca, P. 2000, A\&AS, 144, 469

Samadi, R., \& Goupil, M.-J. 2001, A\&A, 370, 136

Samadi, R., Goupil, M.-J., \& Lebreton, Y. 2001a, A\&A, 370, 147

Samadi, R., Houdek, G., Goupil, M.-J., Lebreton, Y., \& Baglin, A. 2001b, in 1st Eddington Workshop: Stellar Structure and Habitable Planet Finding, ESA SP-485, 87

Samadi, R., Houdek, G., Goupil, M.-J., \& Lebreton, Y. 2002, A\&A, submitted

Soufi, F., Goupil, M. J., \& Dziembowski, W. A. 1998, A\&A, 334, 911 Stein, R. F. 1968, ApJ, 154, 297

Unno, W., Osaki, Y., Ando, H., Saio, H., \& Shibahashi, H. 1989, Nonradial oscillations of stars (Tokyo: University of Tokyo Press) 2nd ed.

Xiong, D. R., \& Deng, L. 2001, MNRAS, 324, 243 